\title{
A New Approximation for the Chi-Square Integral
}

\author{
By H. L. Gray, R. W. Thompson and G. V. McWilliams
}

Abstract. In this paper a new method for estimating the value of an improper integral by a finite sum is introduced. In particular, the method is applied to the Chi-Square integral and proves to be of some value in estimating the value of this integral for values equal or greater than .9 .

I. Introduction. The problem of computing the area, $Q\left(\chi^{2} \mid \nu\right)$, under the right tail of a chi-square distribution is quite important and occurs often in applications. For an even number, $\nu$, of degrees of freedom, the computation of $Q\left(\chi^{2} \mid \nu\right)$ is straightforward (although quite lengthy if $\nu$ is large). For $\nu$ not an even integer, one must use an approximation such as asymptotic or series expansion, normal approximation, or numerical integration. It is well known that most relevant series expansions [1], [5] converge very slowly, often requiring a large number of terms in order to be accurate to only a few significant digits. If only a few significant digits are needed, one can use an asymptotic expansion, but the number of terms used in the series is a function of $\chi^{2}$ and $\nu$; i.e., an approximation using a fixed number of terms from an asymptotic expansion generally has an acceptable accuracy over a limited range of values for $\chi^{2}$ and $\nu$.

In this paper a simple approximation for $Q\left(\chi^{2} \mid \nu\right)$ is developed, and in the process, some new methods for developing approximations are presented. The approximation given here is quite useful for computation on digital computers and for hand calculations when only 3 to $4 \mathrm{D}$ accuracy is needed and if $Q\left(\chi^{2} \mid \nu\right) \leqq .1$.

II. Definitions and Theorems. Let

$$
\gamma(a, z)=\int_{a}^{\infty} e^{-x} x^{z-1} d x, \quad z-1>-1 .
$$

Then

$$
Q\left(\chi^{2} \mid \nu\right)=\frac{\gamma\left(\chi^{2} / 2, \nu / 2\right)}{\Gamma(\nu / 2)} .
$$

For convenience, let $2 u=\chi^{2}$ and $2 v=\nu$. Then $Q\left(\chi^{2} \mid \nu\right)=Q(2 u \mid 2 v)=\gamma(u, v) / \Gamma(v)$.

The approximation we shall derive here uses an approximation for $\gamma(a, z)$ and the truncated Stirling's approximation [1],

$$
\Gamma(z) \simeq e^{-z} z^{z-1 / 2}(2 \pi)^{1 / 2}[1+1 / 12 z] .
$$

In order to derive the approximation for $\gamma(a, z)$, the following definitions and theorems are given. For motivation of these definitions see [2], [3], [4].

Definition 1. If $f \in C^{(1)}$ on $[a, \infty)$ and

Received February 16, 1968, revised July 5, 1968. 


$$
F(t, a)=\int_{a}^{t} f(x) d x \rightarrow L \neq \pm \infty \quad \text { as } t \rightarrow \infty
$$

then

$$
H[F ; t, a] \equiv F(t, a)-f^{2}(t) / f^{\prime}(t), \quad f^{\prime}(t) \neq 0 .
$$

Definition 2. Let $A(t)$ and $B(t)$ be functions of a real variable $t$ such that $A(t)$ $\rightarrow A_{0}$ and $B(t) \rightarrow B_{0}$ as $t \rightarrow \infty . A(t)$ is said to converge more rapidly than $B(t)$ if

$$
\lim _{t \rightarrow \infty}\left(A_{0}-A(t)\right) /\left(B_{0}-B(t)\right)=0 .
$$

Theorem 1. If $\lim _{t \rightarrow \infty} f(t)$ and $\lim _{t \rightarrow \infty} f(t) / f^{\prime}(t)$ exist and the latter is not zero, then $H[F ; t, a]$ converges more rapidly than $F(t, a)$ to $L$.

Proof. Let

$$
\lim _{t \rightarrow \infty} f(t) / f^{\prime}(t)=b \neq 0
$$

then

$$
\begin{aligned}
\lim _{t \rightarrow \infty} \frac{L-H F[; t, a]}{L-F(t, a)} & =1+\lim _{t \rightarrow \infty} \frac{f(t)}{f^{\prime}(t)} \cdot \frac{f(t)}{L-F(t, a)} \\
& =1+b \lim _{t \rightarrow \infty} \frac{f(t)}{L-F(t)}=1-b \cdot \frac{1}{b}=0
\end{aligned}
$$

by L'Hospital's rule. If we let $f(x)=e^{-x} x^{z-1}, z>0$, then

$$
\begin{aligned}
H[F ; t, a] & =\int_{a}^{t} e^{-x} x^{z-1} d x+\frac{e^{-t} t^{z}}{t-z+1} \\
& =-\int_{a}^{t} \frac{(z-1) e^{-x} x^{z-1}}{(x-a+1)^{2}} d x+\frac{e^{-a} a^{z}}{a-z+1} .
\end{aligned}
$$

Note that in (9) the second term is independent of $t$ and that the integrand in (9) is positive and less than the integrand in (8), when $x>z-1+(z-1)^{1 / 2}$. Since (9) converges to the same limit as $F(t, a)$, i.e., $\gamma(a, z)$, as $t \rightarrow \infty$, we can see that for sufficiently large $a$ the integral in (9) does not contribute as much to the limit as does $F(t, a)$. Further, we see that the $H$ transform can be reapplied to the integral in (9) to further increase the speed of convergence. If the integrand is again reduced, then again the resulting integral will contribute less to the desired limit than the previous integral. If this process can be repeated indefinitely, then one may generate a series, dependent only on $a$, which converges to the desired limit and which involves no integration. This is the motivation for the following definition and theorems.

Definition 3. Let $A$ and $B$ be operators defined by

$$
A[f(t)]=f^{2}(t) / f^{\prime}(t)
$$

and

$$
B[f(t)]=f(t)-D_{l} A[f(t)] .
$$

Then we define $H^{n}[F ; t, a]$ by 


$$
H^{n}[F ; t, a]=F(t, a)-\sum_{j=0}^{n-1} A B^{j}[f(t)],
$$

where $B^{j}$ refers to $B$ applied $j$ times and $A B^{0}=A$. An alternate form for (12) is obtained by noting that

$$
\int_{a}^{t} B^{n}[f(x)] d x=F(t, a)+\sum_{j=0}^{n-1} A B^{j}[f(a)]-\sum_{j=0}^{n-1} A B^{j}[f(t)],
$$

which leads to

$$
H^{n}[F ; t, a]=-\sum_{j=0}^{n-1} A B^{j}[f(a)]+\int_{a}^{t} B^{n}(f(x)) a x .
$$

Theorem 2. If

$$
A B^{k} f(t) \rightarrow 0 \quad \text { as } t \rightarrow \infty, \quad k=0,1, \cdots, n-1,
$$

then

$$
\lim _{t \rightarrow \infty} H^{n}[F ; t, a]=L .
$$

Proof. The proof follows immediately from (12).

Theorem 3. If

$$
\lim _{n \rightarrow \infty} \int_{a}^{\infty} B^{n}(f(t)) d t=0
$$

and

$$
\lim _{t \rightarrow \infty} A B^{k}(f(t))=0, \quad k=0,1, \cdots
$$

then

$$
\int_{a}^{\infty} f(x) d x=-\sum_{j=0}^{\infty} A B^{j}(f(a)) .
$$

Proof. The proof follows immediately from (13).

Theorems 2 and 3 indicate that under certain conditions the repeated application of $H$ will, in fact, produce a series which converges to the desired limit. This immediately suggests that the terms of the series derived after $n$ applications of the $H$ transform may be a good approximation for the original improper integral. If the approximation is good for small $n$, then the method may produce a worthwhile approximation function. This is, in fact, the case for $\gamma(a, z)$.

III. Derivation of the Approximation. Let $f(t)=e^{-t} t^{z-1}$ and

$$
\gamma_{n}(a, z)=-\sum_{j=0}^{n} A B^{j} f(a) .
$$

Then, letting $p=a-z+1$,

$$
\begin{aligned}
& \gamma_{0}(a, z)=e^{-a} a^{z} / p, \\
& \gamma_{1}(a, z)=\frac{e^{-a} a^{z}}{p}\left(1-\frac{z-1}{p^{2}+2 a}\right),
\end{aligned}
$$




$$
\begin{aligned}
& \gamma_{2}(a, z)=\frac{e^{-a} a^{2}}{p}\left(1-\frac{z-1}{p^{2}+2 a}\right. \\
& \left.+\frac{(z-1)\left[(z-1) p^{2}+2 a^{2}\right]^{2}}{p\left(p^{2}+2 a\right)\left[(z-1) p^{6}-2 a p^{5}+4 a(z-2) p^{4}+8 a^{2} p^{3}-16 a^{3} p^{2}-8 a^{4}\right]}\right) .
\end{aligned}
$$

As can be seen, the calculation of further terms becomes quite difficult. Because of its simplicity and because $\gamma_{2}$ yields very little more accuracy than $\gamma_{1}$ in the range of values of interest here, $Q(2 u \mid 2 v) \leqq 0.1, \gamma_{1}$ is used in the approximation. Thus

$$
Q(2 u \mid 2 v)=\frac{e^{-u} u^{v}}{p \Gamma(v)}\left(1-\frac{v-1}{p^{2}+2 u}\right) .
$$

One difficulty inherent in many methods, including (22), of computing $\chi^{2}$ probabilities is that the differences of the magnitudes of $e^{-u}, u^{v}$, and $\Gamma(v)$ are often extreme. One method of alleviating this problem and at the same time avoiding the calculation of $\Gamma(v)$, is by use of (3), yielding the quantity

$$
C\left(\chi^{2}, \nu\right)=\frac{e^{-(u-v)}}{p(2 \pi)^{1 / 2}}\left(\frac{u}{v}\right)^{v}\left(1-\frac{v-1}{p^{2}+2 u}\right)\left(\frac{12 v^{3 / 2}}{12 v+1}\right),
$$

where $2 u=\chi^{2}, 2 v=\nu$, and $p=u-v+1$, as an approximation of $Q\left(\chi^{2} \mid \nu\right)$. It is this latter approximation that we will investigate further.

TABLE 1

Errors in $C\left(\chi^{2}, \nu\right)$ and $Q_{3}\left(\chi^{2} \mid \nu\right)$

\begin{tabular}{rlrr}
\hline$\nu$ & $Q$ & $C\left(\chi^{2}, \nu\right)-Q$ & $Q_{3}\left(\chi^{2} \mid \nu\right)-Q$ \\
\hline \hline 1 & .1 & -.00105 & .03035 \\
1 & .05 & -.00028 & .00620 \\
1 & .01 & -.00002 & .00029 \\
2 & .1 & .00010 & .00010 \\
2 & .05 & .00005 & .00005 \\
2 & .01 & .00001 & .00001 \\
5 & .1 & .00054 & .00010 \\
5 & .05 & .00013 & .00001 \\
5 & .01 & .00000 & .00000 \\
10 & .1 & .00058 & -.00302 \\
10 & .05 & .00010 & -.00107 \\
10 & .01 & .00000 & -.00011 \\
30 & .1 & .00038 & -.02007 \\
30 & .05 & .00004 & -.00811 \\
30 & .01 & -.00001 & -.00109 \\
100 & .1 & .00007 & -.04464 \\
100 & .05 & -.00022 & -.01975 \\
100 & .01 & -.00004 & -.00313 \\
250 & .1 & -.00001 & -.06645 \\
250 & .05 & -.00033 & -.02787 \\
250 & .01 & -.00006 & -.00537 \\
500 & .1 & -.00018 & -.06975 \\
500 & .05 & -.00043 & -.03761 \\
500 & .01 & -.00007 & -.00586 \\
\hline
\end{tabular}


IV. Comparison with Other Estimators. In order to examine the accuracy of $C^{\prime}\left(\chi^{2}, \nu\right)$ as an approximation for $Q\left(\chi^{2} \mid \nu\right), C\left(\chi^{2}, \nu\right)$ was computed for $\nu=1,2,5,10$, $20,30,100,250,500$, using values of $\chi^{2}$ which yield $Q\left(\chi^{2} \mid \nu\right) \simeq 0.1,0.05$, and 0.01 . The results shown in Table 1 indicate that, except at $\nu=1$ for $Q\left(\chi^{2} \mid \nu\right)=0.1$, $C\left(\chi^{2}, \nu\right)$ is accurate to at least $3 \mathrm{D}$.

For the sake of comparison, consider the series expansion for $Q\left(\chi^{2} \mid \nu\right)$,

$$
Q\left(\chi^{2} \mid \nu\right)=1-\frac{e^{-\chi^{2}}\left(\chi^{2}\right)^{(\nu / 2)+1}}{\Gamma((\nu+1) / 2)}\left\{1+\frac{\chi^{2}}{\nu / 2+2}+\frac{\left(\chi^{2}\right)^{2}}{(\nu / 2+2)(\nu / 2+3)}+\cdots\right\}
$$

as a method of estimating $Q\left(\chi^{2} \mid \nu\right)$.

Using (24), one can compute $Q\left(\chi^{2} \mid \nu\right)$ to any desired accuracy, but the number of terms required can be large; e.g., after 14 terms, (24) yields a less accurate estimate of $Q(8 \mid 4)$ than does $C(8,4)$, while for $\nu=100$ and $\chi^{2}=118.498$, (24) is less accurate at 40 terms than is $C(118.498,100)$. Another method of estimating $Q\left(\chi^{2} \mid \nu\right)$ is by use of the asymptotic series. The problem of determining how many terms of an asymptotic series to use in making an estimate of the value of a function is well known. In order to compare $C\left(\chi^{2}, \nu\right)$ with a truncated asymptotic series, the first three terms of the asymptotic series for $Q\left(\chi^{2} \mid \nu\right)$ were used in conjunction with (3), yielding

$$
Q_{3}\left(\chi^{2} \mid \nu\right)=\frac{e^{-(u-v)}}{(2 \pi)^{1 / 2}}\left(\frac{u}{v}\right)^{v-1}\left(1+\frac{(v-1)}{u}+\frac{(v-1)(v-2)}{u^{2}}\right)\left(\frac{12 \sqrt{ } v}{12 v+1}\right) .
$$

$Q_{3}\left(\chi^{2} \mid \nu\right)$ yields a good estimate of $Q\left(\chi^{2} \mid \nu\right)$ and is of the same order of complexity as $C\left(\chi^{2}, \nu\right)$. As shown in Table 1 , however, $C\left(\chi^{2}, \nu\right)$ generally yields a much better estimate than does $Q_{3}\left(\chi^{2} \mid \nu\right)$.

Texas Technological College

Lubbock, Texas 79409

LTV Electrosystems

Greenville, Texas 75401

1. M. Abramowitz \& I. A. Stegun (Editors), Handbook of Mathematical Functions, with Formulas, Graphs and Mathematical Tables, Nat. Bur. Standards, Appl. Math. Series, 55, Superintendent of Documents, U. S. Government Printing Office, Washington, D. C., 1964, p. 257; 3rd printing with corrections, 1965. MR 29 \#4914; MR 31 \#1400.

2. H. L. Gray \& T. A. Atchison, "Nonlinear transformations related to the evaluation of improper integrals," SIAM J. Numer. Anal., v. 4, 1967, pp. 363-371.

3. H. L. Gray \& T. A. A tchison, "Nonlinear transformations related to the evaluation of improper integrals. II," SIAM J. Numer. Anal., v. 5, 1968, pp. 451-459.

4. H. L. Gray \& W. R. Schucany, "On the evaluation of distribution functions," J. Amer. Statist. Assoc., v. 63, 1968, pp. 715-720.

5. R. TakenaGa, "On the evaluation of the incomplete gamma function," Math. Comp., v. 20, 1966, pp. 606-610. MR 34 \#3758. 